\title{
Real-World Experience with Oritavancin Therapy in Invasive Gram-Positive Infections
}

\author{
Cassie L. Stewart · Michelle S. Turner · Jeremy J. Frens • \\ Cynthia B. Snider $\cdot$ Jordan R. Smith
}

Received: February 16, 2017 / Published online: April 6, 2017

(C) The Author(s) 2017. This article is an open access publication

\begin{abstract}
Introduction: Oritavancin is a novel lipoglycopeptide approved for acute bacterial skin and skin structure infections. The pharmacokinetic profile and convenient one-time dosing make oritavancin an enticing option for other serious Gram-positive infections requiring prolonged treatment courses. Unfortunately, data for using oritavancin in these populations are limited.

Methods: We report ten cases of oritavancin use for invasive Gram-positive infections in our health system, and provide a review of the currently available literature regarding oritavancin therapy for invasive infections.

Results: Among the ten patients who received oritavancin, the most common infection was methicillin-susceptible Staphylococcus aureus (MSSA) bacteremia $(n=5,50 \%)$. Other indications for oritavancin use included methicillin-resistant $S$. aureus (MRSA) bursitis $(n=1$,
\end{abstract}

Enhanced content To view enhanced content for this article go to http://www.medengine.com/Redeem/ 3708F0602569295B.

C. L. Stewart · M. S. Turner · J. J. Frens .

C. B. Snider · J. R. Smith

Moses Cone Hospital, Cone Health, Greensboro,

NC, USA

\section{J. R. Smith ( $\square)$}

Fred Wilson School of Pharmacy, High Point

University, High Point, NC, USA

e-mail: jsmith5@highpoint.edu
10\%), group B streptococcal bacteremia with native tricuspid valve infective endocarditis $(n=1,10 \%)$, coagulase-negative staphylococcal bacteremia $(n=1,10 \%)$, MSSA deep tissue infection $(n=1,10 \%)$, and enterococcal bacteremia $(n=1,10 \%)$. Oritavancin was well tolerated, and $7 / 10$ (70\%) patients were successfully treated.

Conclusion: Oritavancin is a potential option for patients with invasive Gram-positive infections. Further study is warranted.

Keywords: Bacteremia; Extended duration; Lipoglycopeptide; OPAT; Outpatient parenteral antimicrobial therapy; Staphylococci; Staphylococcus aureus

\section{INTRODUCTION}

Gram-positive pathogens are a leading cause of serious community-acquired and healthcare-associated infections [1]. Staphylococcus aureus is the most common pathogen isolated among healthcare-associated infections, and in the United States, roughly half of all $S$. aureus isolated from the bloodstream are now resistant to methicillin [2]. Methicillin-resistant $S$. aureus (MRSA) is independently associated with increased morbidity and mortality [3, 4]. Bloodstream infections with $S$. aureus are rife with complications such as osteomyelitis and endocarditis, and treatment courses tend to 
involve daily, intravenous (IV) antibiotic administration and last 6 weeks or more, which is a challenge for both patients and providers [5]. Enterococci, another group of frequently isolated hospital-acquired pathogens, can also cause serious and potentially resistant infections [2]. Over the past several decades, mutations in the vanA and $v a n B$ genes have caused vancomycin-resistant strains of Enterococcus faecalis and E. faecium to emerge. The prevalence of vancomycin-resistant enterococci has increased substantially over the last several years, and treatment options are limited [2].

Vancomycin has been a mainstay of therapy for Gram-positive infections, especially MRSA, but long-term outpatient parenteral antimicrobial therapy (OPAT) with vancomycin is complicated due to frequent dosing and the need for therapeutic drug monitoring to prevent potential toxicities [6]. Some patients are not candidates for prolonged intravenous antibiotics, including those likely to abuse a vascular access system and those unable to adhere to an OPAT regimen [7]. Furthermore, approximately 10\% of OPAT regimens must be stopped prematurely due to adverse reactions, which may complicate the course of treatment and require a longer treatment duration with other antibiotics [7]. Treatment of Gram-positive infections, especially with $S$. aureus, presents a clinical challenge in need of creative therapeutic approaches.

Oritavancin is a novel lipoglycopeptide approved by the Food and Drug Administration for the treatment of acute bacterial skin and skin structure infections (ABSSSIs) [8]. It is structurally similar to vancomycin, but possesses an additional hydrophobic tail that alters both its pharmacokinetic and antimicrobial properties [9]. It possesses three distinct mechanisms of action, inhibiting both transpeptidation and transglycosylation, causing disruption of cell wall synthesis, and also disrupting the cell membrane through membrane binding of its hydrophobic tail [9]. Unlike many other agents approved for ABSSSIs, oritavancin is given as a one-time dose of $1200 \mathrm{mg}$ IV infused over $3 \mathrm{~h}$. The single dose is sufficient for ABSSSIs due to the initial peak serum concentration of $140 \mathrm{mg} / \mathrm{L}$ and prolonged terminal half-life, which ranges from 245 to $393 \mathrm{~h}$ ( 10-17 days) [10, 11]. Pharmacokinetic data demonstrate that, on average, oritavancin maintains concentrations in several body compartments above the $\mathrm{MIC}_{90}$ threshold for at least a month from initial dosing [10]. Oritavancin is active against a variety of Gram-positive species, and surveillance data from 2008 to 2012 of over $9000 \mathrm{~S}$. aureus isolates demonstrate a minimum inhibitory concentration (MIC) $)_{90}$ of only $0.06 \mathrm{mg} / \mathrm{L}$ [12]. Among the 205 isolates with vancomycin MICs of $2 \mathrm{mg} / \mathrm{L}$ and the 100 isolates with daptomycin MICs of $\geq 1 \mathrm{mg} / \mathrm{L}$ within this study, the oritavancin $\mathrm{MIC}_{90}$ was $0.12 \mathrm{mg} / \mathrm{L}$, and only 13 of these 305 (4\%) isolates possessed oritavancin MICs $>0.12 \mathrm{mg} / \mathrm{L}$, the current FDA breakpoint for susceptibility [12]. From 2013 to 2014, when oritavancin was tested against 780 invasive community-acquired and 218 invasive healthcare-associated $S$. aureus isolates from blood culture specimens, all $S$. aureus were susceptible to oritavancin and the $\mathrm{MIC}_{50 / 90}$ values were $0.03 / 0.06 \mathrm{mg} / \mathrm{L}$, respectively [13]. These MICs were consistent regardless of oxacillin susceptibility or multi-drug resistance phenotypes. Nearly all isolates were susceptible to vancomycin, daptomycin, and linezolid, but oritavancin $\mathrm{MIC}_{50}$ and $\mathrm{MIC}_{90}$ values were eightfold lower than daptomycin and 16- to 32-fold lower than vancomycin or linezolid [13]. Oritavancin has also shown potent in vitro activity against methicillin-resistant coagulase-negative staphylococci (CoNS) with $\mathrm{MIC}_{50}$ and $\mathrm{MIC}_{90}$ of 0.06 and $0.12 \mathrm{mg} / \mathrm{L}$, respectively [14]. Oritavancin also possesses in vitro activity against enterococci, achieving a $\mathrm{MIC}_{90}$ of $0.06 \mathrm{mg} / \mathrm{L}$ against VRE in one surveillance study [15]. Although this agent is currently indicated solely for ABSSSIs, the therapeutic potential for disease states necessitating prolonged therapy, such as Gram-positive bacteremia and endocarditis, is enticing.

Our health system has used oritavancin in several cases of invasive Gram-positive infections where IV antibiotics were not considered a safe option, the patient refused IV antibiotic therapy, or antibiotics such as cefazolin, vancomycin, or daptomycin were not feasible. 
Currently, oritavancin is not on our health system's formulary, and the infectious diseases (ID) physicians are the only providers prescribing oritavancin. Here, we document several cases in an effort to describe the efficacy and safety of oritavancin for serious Gram-positive indications other than ABSSSIs, and provide a review of the current available literature regarding off-label oritavancin therapy.

\section{METHODS}

\section{Study Design and Population}

This was a retrospective, observational chart review of adult ( $\geq 18$ years of age) patients using electronic medical records at Cone Health, a 1254-bed, five-hospital healthcare system based in Greensboro, North Carolina, USA. Eligible patients received at least one dose of oritavancin for an indication other than ABSSSI from April to December 2015. April was chosen as the first month included in the study, because it was the first month that a dose of oritavancin was ordered in our health system. Patients were identified by VigiLanz $^{\mathrm{TM}}$ decision support software.

\section{Compliance with Ethics Guidelines}

This was a retrospective study reviewing chart data, therefore no new studies with human or animal subjects were performed by any of the authors. A waiver of informed consent was obtained from the Cone Health Institutional Review Board.

\section{Data Collection}

Patient demographics, comorbid conditions, primary infection site, microbiological data, antimicrobial therapy employed in treatment, length of hospital stay, and overall clinical outcome as judged by the investigators were collected. With respect to oritavancin, the indication, number of doses administered, and adverse effects were collected.

\section{Safety Assessment}

Safety data included adverse event reporting by the prescribing physician and follow-up with patients after oritavancin administration.

\section{Definitions}

Patient outcomes were categorized as clinical cure or failure. Clinical cure was defined as resolution of all clinical signs and symptoms of infection, which included resolution of fever, normalization of white blood cell count and erythrocyte sedimentation rate, with no additional infection-related hospital admissions, no additional antibiotic therapy required for the indication initially treated with oritavancin, and infection clearance with sterile blood cultures where indicated. Failure was defined as worsening of current infection or new/recurrent signs and symptoms of infection requiring either a change or addition of antibiotic therapy. Patients lost to follow-up were classified as not clinically evaluable.

\section{RESULTS}

A total of ten patients received off-label oritavancin during the pre-specified study period at our health system. Table 1 summarizes the clinical course and outcomes for all patients. The median patient age was 46 (range 2966) years. The most common infection leading to off-label use of oritavancin was methicillin-susceptible $S$. aureus (MSSA) bacteremia $(n=5,50 \%)$. Other indications included MRSA bursitis $(n=1,10 \%)$, group B streptococcal bacteremia with native tricuspid valve endocarditis $\quad(n=1,10 \%), \quad$ coagulase-negative staphylococcal bacteremia $(n=1,10 \%)$, MSSA deep tissue infection $(n=1,10 \%)$, and enterococcal bacteremia $(n=1,10 \%)$. All but one patient received a one-time 1200-mg dose of oritavancin. There were a number of antibiotics used prior to initiation of oritavancin therapy, the most common being vancomycin (9 patients, 90\%) and cefazolin (6 patients, 60\%). All patients received oritavancin following 


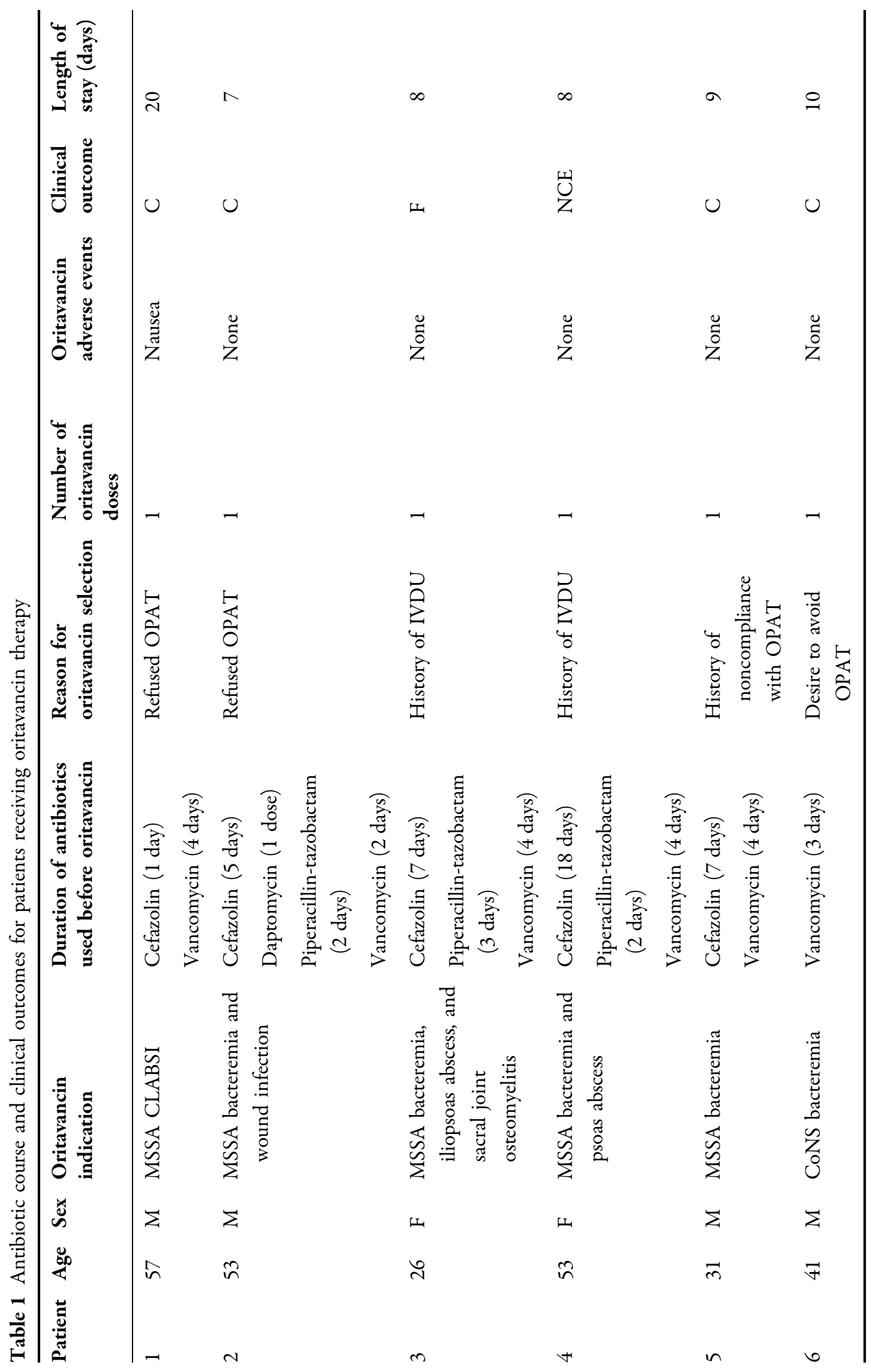




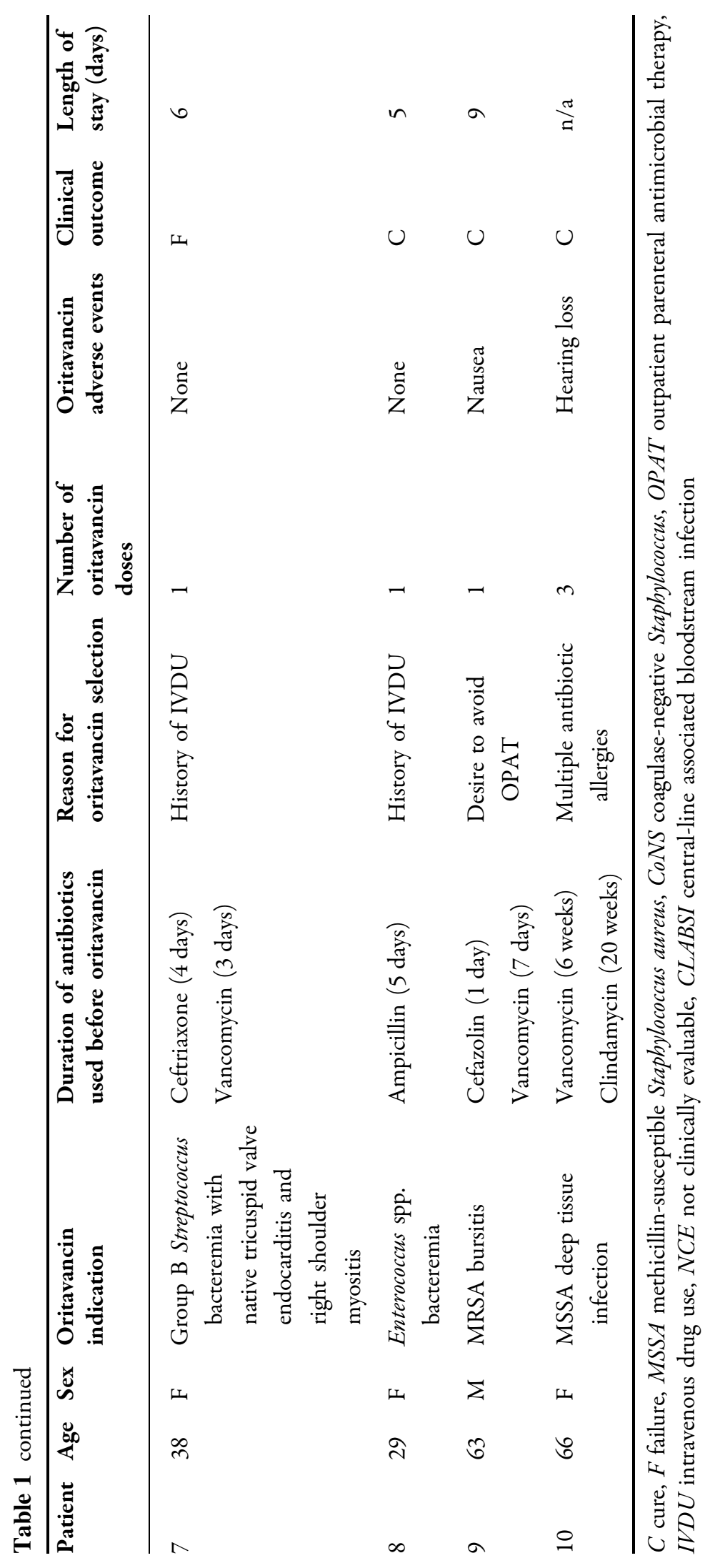


different courses of standard antibiotic therapy for continued clearance of the initial infection. Safety data revealed that oritavancin was generally well tolerated. One patient developed nausea during administration of oritavancin that quickly resolved after the infusion was completed. Another patient, who received three doses of oritavancin, experienced hearing loss documented by an audiologist, a heretofore unseen reaction to oritavancin. However, this patient also recently completed a course of vancomycin therapy. Contact was attempted with all patients after receiving oritavancin by either follow-up in our outpatient infectious diseases clinic or by telephone. Seven patients (70\%) were clinically cured of their initial infection after receiving oritavancin, two patients (20\%) failed oritavancin therapy, and one patient $(10 \%)$ was unable to be contacted for follow-up

\section{CASE REPORTS}

The ten patients who received oritavancin are discussed below.

\section{Patient 1}

A 57-year-old male with a history of rheumatic heart disease status post-aortic and mitral valve replacement, severe congestive heart failure, and chronic kidney disease was admitted with shortness of breath, bilateral lower extremity edema, and abdominal distention. Due to bleeding complications and loss of central line access, he had a peripherally inserted central catheter (PICC) placed during his treatment course. One week later, he complained of intense pain, swelling, and redness around the PICC placement site. The PICC line was removed, blood and catheter tip cultures were obtained, and he was started on vancomycin. Preliminary blood cultures revealed $S$. aureus. An ID physician consultation was sought, where cefazolin was added in addition to vancomycin. After susceptibilities revealed MSSA (resistant to penicillin), the patient's regimen was narrowed to cefazolin. A transesophageal echocardiogram (TEE) was obtained which revealed no evidence of infective endocarditis on his native or prosthetic valves. Due to complications he experienced with his central line and PICC, he was reluctant to have a second PICC line placed again for OPAT. He received one dose of oritavancin after 1 day of cefazolin and 4 days of vancomycin, and after repeat blood cultures had been negative for $48 \mathrm{~h}$. He was discharged the next day, and at follow-up in our outpatient ID clinic, a third set of blood cultures was collected and remained sterile, approximately 22 days after his oritavancin dose.

\section{Patient 2}

A 53-year-old male with an extensive history including paraplegia, chronic ischial decubitus ulcers with osteomyelitis, chronic left knee ulcers with osteomyelitis, epididymitis, and recurrent staphylococcal and streptococcal bacteremia was admitted with severe scrotal drainage and edema. He was septic on admission and started on broad spectrum antibiotics with vancomycin and piperacillin-tazobactam. Urine cultures drawn on admission were positive for Klebsiella pneumoniae (resistant to ampicillin and intermediate to nitrofurantoin), and blood cultures drawn on admission were positive for MSSA (susceptible to all antibiotics tested). ID was consulted, and after 2 days of vancomycin and piperacillin-tazobactam, he received a one-time dose of daptomycin but was ultimately switched to cefazolin monotherapy. A transthoracic echocardiogram (TTE) revealed no evidence of vegetations, and he refused to have a TEE performed. He has a history of noncompliance and refusal of IV antibiotics and again refused IV antibiotics and placement into a skilled nursing facility on this admission. Due to this, the ID physician prescribed a dose of oritavancin after he received 5 days of cefazolin and after repeat blood cultures were negative. He was scheduled to have another dose of oritavancin 2 weeks after his first dose, but failed to show up to his appointments at our infusion center or ID clinic. Upon contact with the patient after discharge, he noted traveling to New York to receive a skin flap for his chronic 
decubitus ulcers, but no hospital admissions, no further infections, and no other antibiotics needed after the one-time oritavancin dose.

\section{Patient 3}

A 26-year-old female with active intravenous drug use (IVDU) and no other concomitant medical conditions was admitted with fever, chills, and acute low back pain. Blood cultures obtained on admission were positive for MSSA (resistant to penicillin). Imaging of her lumbar spine and pelvis revealed that she had an iliopsoas abscess and sacroiliac joint infection. Aspirate of the iliopsoas abscess was also culture positive for MSSA. Dual therapy was initiated with vancomycin and cefazolin, which was subsequently narrowed to cefazolin when susceptibility data became available. Her TEE was negative for endocarditis, as well as her repeat blood cultures on cefazolin. She was determined to not be a candidate for OPAT due to active IVDU. She received one dose of oritavancin once negative blood cultures were finalized and was discharged on oral clindamycin. At outpatient follow-up 6 weeks after discharge, she reported non-adherence to oral clindamycin due to inability to afford medication, and complained of continued hip pain, night sweats, fever, and chills. Antibiotics were re-instituted at this time, with a 1-month course of cephalexin and plans for repeat imaging. Three weeks into her cephalexin course, she presented to our emergency department (ED) where she reported increased hip pain and fevers. Repeat imaging showed worsening sacroiliac infection with surrounding osteomyelitis. The orthopedic surgeon consultation recommended medical management rather than irrigation and debridement of her sacroiliac joint due to the location and complicated nature of her infection. She was started on a course of vancomycin, and blood cultures obtained on admission remained sterile throughout the hospitalization. Vancomycin was discontinued by ID who then recommended joint aspiration by interventional radiology. There was no growth on the joint aspiration culture, and she was discharged on a 3-month course of sulfamethoxazole/trimethoprim and rifampin. She was scheduled for a follow-up in our outpatient ID clinic 2-3 weeks after discharge but was lost to follow-up. She subsequently returned back to our ED 2 months after her last hospitalization with intense hip pain and fevers. Per ID, she was started on cefazolin for 6-8 weeks with a repeat MRI in 4 weeks. A TTE revealed no evidence of vegetations. Blood cultures remained sterile throughout the hospital stay, but, due to continued fevers while on antibiotics and a new systolic heart murmur found on examination, a TEE was obtained, which revealed a small aortic valve vegetation consistent with infective endocarditis. Although her subsequent blood cultures remained sterile, she most likely never cleared her sacroiliac joint infection, which was thought to be the sequelae of initial MSSA bacteremia, and nidus for development of infective endocarditis.

\section{Patient 4}

A 53-year-old female with active IVDU, hypertension, and bipolar disorder was admitted with a 2-week history of right hip/buttock pain surrounding the site where she injects heroin. She was started on broad spectrum antibiotics with vancomycin and piperacillin-tazobactam. Imaging of her right hip showed extensive edema surrounding the pelvis with a psoas abscess and possible early osteomyelitis. Blood cultures revealed $S$. aureus, so an ID consult was sought and after 2 days of vancomycin and piperacillin-tazobactam, her antibiotics were changed to vancomycin and cefazolin. A TTE revealed no evidence of vegetations, and she refused a TEE. Susceptibilities revealed MSSA (susceptible to all antibiotics tested), and her antibiotics were subsequently narrowed to cefazolin. Orthopedic surgeons did not feel surgical debridement of her right hip was necessary, and they recommended follow-up imaging in 1 month. Due to the patient's active IVDU, she was thought not to be a candidate for OPAT. After her repeat blood cultures were negative, she was discharged to a skilled nursing facility with IV cefazolin for at least 6 weeks 
with follow-up in the outpatient ID clinic in 2-3 weeks. She left the skilled nursing facility against medical advice only 8 days after being discharged from the hospital with a PICC line still in place. She followed up with ID the next day in the outpatient ID clinic where she was prescribed a dose of oritavancin after approximately 18 days of cefazolin. She failed to obtain repeat imaging after her oritavancin dose. Since her dose of oritavancin, she has failed to follow-up in the ID clinic, and we were unable to reach her by telephone. She has had no other hospital admissions at our health system.

\section{Patient 5}

A 31-year-old male with a complicated history including Job syndrome, aspergilloma on chronic posaconazole, hypertension, anxiety, muscle spasms, and anemia was admitted with left lower leg swelling and pain. He also has an extensive infectious disease-related history including cryptococcal meningitis as an infant, multiple intrathoracic and intraabdominal infections, and recurrent MSSA and MRSA bacteremia. Imaging on admission showed possible cellulitis with no evidence of an abscess or osteomyelitis, and blood cultures revealed $S$. aureus. He was started on vancomycin, and a TEE revealed no evidence of vegetations. He has a history of noncompliance with outpatient antibiotic therapy with undetectable vancomycin levels, so he was deemed not a candidate for OPAT. Susceptibility data revealed MSSA (resistant to clindamycin and erythromycin), and he was prescribed a dose of oritavancin after receiving 4 days of vancomycin and 7 days of cefazolin and after repeat blood cultures were negative. Repeat blood cultures 9 days after his oritavancin dose were also negative.

\section{Patient 6}

A 41-year-old male with a history of chronic back pain, pseudoseizures, vertebral osteomyelitis with group B streptococcal infection, fungemia, depression, and polysubstance abuse was admitted with back pain and suicidal thoughts. Blood cultures obtained on admission were positive for coagulase-negative Staphylococcus (resistant to clindamycin, erythromycin, levofloxacin, oxacillin, penicillin, rifampin, tetracycline, and trimethoprim-sulfamethoxazole; susceptible to gentamicin and vancomycin only). The patient was started on vancomycin. After repeat blood cultures had been negative for $72 \mathrm{~h}$ and a TTE was negative for endocarditis, the patient was given a one-time dose of oritavancin after 3 days of vancomycin to preclude any need for OPAT due to the patient's extensive psychiatric history. Per ID, no follow-up was needed in the outpatient ID clinic. Upon follow-up with the patient by phone call, he denied any additional infection-related hospital admissions or antibiotics needed for his infection.

\section{Patient 7}

A 38-year-old female with active IVDU and no other chronic medical conditions was admitted with diaphoresis, right index finger pain, and shoulder pain. Imaging of her right shoulder showed myositis without evidence of abscess, and blood cultures on admission revealed Streptococcus agalactiae (resistant to clindamycin and erythromycin). Her initial work-up with TTE revealed that she had native tricuspid valve infective endocarditis. She was started on intravenous vancomycin therapy and subsequently narrowed to intravenous ceftriaxone. An evaluation by cardiothoracic surgery recommended medical management only due to lack of evidence of right-sided heart failure or tricuspid regurgitation. She was deemed to not be a candidate for OPAT due to active IVDU. The ID physician prescribed one dose of oritavancin prior to discharge after 3 days of vancomycin and 4 days of ceftriaxone, and after repeat blood cultures had been negative for $48 \mathrm{~h}$. Upon contact with the patient after discharge, she revealed that she had moved out of state and subsequently required valve replacement surgery 3 months after her oritavancin dose. Due to the need for surgical intervention and hospital readmission, she was deemed a clinical failure. 


\section{Patient 8}

A 29-year-old female with active IVDU and no other chronic medical conditions was admitted with a left hand abscess. Her hand infection had started over 4 weeks prior to hospital admission, where she had an incision and drainage by a local orthopedic surgeon in the ambulatory setting. Upon admission, the patient received incision and drainage procedure of her left hand abscess with specimen sent for culture. Her wound culture isolated microaerophilic streptococcus, and she was discharged after surgery with oral doxycycline. One day after discharge, one of two blood cultures obtained during her admission grew Enterococcus species, and she was contacted to return to the hospital. She was readmitted for enterococcal bacteremia but also complained of fever, chills, and continued hand pain. The enterococcus isolate from blood cultures was vancomycin- and ampicillin-sensitive; thus, the patient was initiated with intravenous ampicillin. A TEE was negative for endocarditis. Given her ongoing IVDU, there was concern she would abuse her PICC line needed for OPAT. She wanted to leave against medical advice, so her ID physician prescribed one dose of oritavancin after 5 days of ampicillin and after repeat blood cultures had been negative for $48 \mathrm{~h}$. She did not keep her follow-up ID appointment. On a follow-up phone call, she denied any other hospital admissions for the infection and any other antibiotic use after oritavancin.

\section{Patient 9}

A 63-year-old male with a history of hypertension was admitted with a 1-week history of left knee pain. Imaging revealed soft tissue swelling with septic prepatellar bursitis. Knee aspiration was performed in the emergency department yielding gross purulence, and he was started on vancomycin. Orthopedic surgery initially elected to treat the patient with antibiotics only, but he spiked a high fever and had continued leukocytosis despite antibiotics, so incision and drainage of the knee was performed. Cultures of the aspirate grew MRSA (resistant to erythromycin, oxacillin, and penicillin), and the initial plan was to continue IV antibiotic therapy with vancomycin while hospitalized and transition to oral doxycycline on discharge for a total of 3 weeks of antibiotics. He required another incision and drainage with closure 5 days after his first surgery. At that time, it was decided by ID to give him a one-time dose of oritavancin to preclude any need for antibiotics post-discharge, and after he had received 1 day of cefazolin and 7 days of vancomycin. He did endorse severe nausea while the oritavancin dose was infusing that quickly resolved after completion. When he followed-up in our outpatient ID clinic 4 months after his hospitalization, his infection had resolved with no further symptoms, drainage, or need for further antibiotics or hospitalizations.

\section{Patient 10}

A 66-year-old female with a history of hypertension, depression, previous MRSA infection, gastric bypass, and gastroesophageal reflux disease sustained a complicated work-related injury, which included right wrist and humerus fracture managed by open reduction and internal fixation. Post-operative day 10, she developed a deep shoulder joint abscess with MSSA (susceptible to all antibiotics tested) requiring a second surgery with irrigation and debridement and hardware removal. She was treated initially with a 6-week course of intravenous vancomycin followed by oral clindamycin. Her shoulder infection showed improvement after 5 weeks of clindamycin, but then she was found to have developed a massive left thigh hematoma that required surgical evacuation with debridement of necrotic tissue and wound vacuum placement. She was continued on oral clindamycin for an additional 7 weeks due to suspected staphylococcal infection. She required multiple surgical debridements, and antibiotics were stopped since the wound bed appeared to be healing with no signs of infection. After 4 weeks of being off antibiotics, the patient's leg wound appeared to have increasing induration and drainage, thus clindamycin was reinitiated. MSSA was isolated from a wound culture obtained at this time, and 
susceptibilities now revealed clindamycin resistance. Oritavancin was chosen to treat her deep tissue infection since the patient reported allergies to cephalexin (anaphylaxis), tetracyclines (anaphylaxis), and sulfa antibiotics (hives), which limited antibiotic treatment selection. She received one dose of oritavancin after 20 weeks of clindamycin and tolerated the dose without difficulty. On follow-up, she was deemed to have a suboptimal response to her first dose of oritavancin by evidence of continued wound drainage and elevated erythrocyte sedimentation rate (ESR), thus a second dose was administered, which was 19 days after receiving the first dose. During a follow-up visit, the patient reported new-onset hearing loss and was referred for audiology testing. She had received her final dose of oritavancin 14 days after her second dose for a total of three doses in a 33-day period. Evaluation by audiology was conducted after she received her third dose where the patient was diagnosed with moderate high frequency sensorineural hearing loss. At outpatient follow-up 3 weeks after her third oritavancin dose, she was clinically cured and her inflammatory markers had normalized. The patient reported some mild improvement in hearing loss but still had tinnitus.

\section{DISCUSSION AND REVIEW OF LITERATURE}

Invasive Gram-positive infections, especially with $S$. aureus, are associated with high morbidity and mortality and often lead to complications, such as infective endocarditis [2]. Alternative options with convenient dosing, ideal pharmacokinetics, and desirable safety profiles are needed for patients who cannot tolerate or use outpatient intravenous antibiotics, such as vancomycin. Oritavancin is a novel lipoglycopeptide that is currently FDA-approved for treatment of ABSSSIs. Its convenient one-time dosing and long half-life, low MICs for Gram-positive organisms, and absence of a need for therapeutic drug monitoring make it an appealing choice for other serious Gram-positive infections. This case series describes the outcomes of patients treated with oritavancin for off-label indications that could not be managed with standard therapy. These cases supplement the limited available data demonstrating the use of oritavancin in patients with Gram-positive infections other than ABSSSIs.

Two patients who received oritavancin for invasive Gram-positive infections failed therapy. Patient 3 illustrated a complex course receiving multiple antibiotics for her sacroiliac septic arthritis and osteomyelitis. After her oritavancin dose, she was eventually readmitted several times for worsening infection. She reported noncompliance with several outpatient antibiotic regimens due to inability to afford medication, which could have caused re-seeding of her joint infection into her bloodstream and eventually causing endocarditis. Another explanation could be that the initial TEE obtained on her first admission was a false negative. The patient eventually had irrigation and debridement of her hip 6 months after her initial infection with placement of antibiotic beads and was continued on chronic suppressive therapy with cephalexin.

Patient 7 was also deemed an oritavancin failure. She had evidence of a large tricuspid valve vegetation on initial TTE. Due to no evidence of right-sided heart failure or tricuspid valve regurgitation, cardiothoracic surgery decided on medical management only. She was discharged in stable condition after her onetime dose of oritavancin. She never followed-up in our outpatient ID clinic, but on a follow-up phone call, she revealed that she had moved away and become sick again requiring readmission to a hospital. During that hospitalization, she required heart valve replacement due to ongoing endocarditis and her deteriorating condition.

Patient 10, who received 6 weeks of IV vancomycin followed by three doses of oritavancin, developed high-frequency hearing loss. Although not common, vancomycin has been associated with ototoxicity. According to a case series by Mellor et al., two patients who received vancomycin developed tinnitus and vertigo that quickly resolved after discontinuation of vancomycin [16]. To date, there have been no case reports of ototoxicity with oritavancin 
therapy. Our patient did not have previous audiology testing before receiving antibiotics. She noticed intermittent ringing in her ears possibly during her vancomycin therapy, but did not mention this to her physician until after the first oritavancin dose. We are not sure if her hearing loss was due to her initial vancomycin, her subsequent oritavancin, or simply age-related. The audiology report also states that it is difficult to say if it is due to antibiotic therapy, but the onset of symptoms during therapy is curious. The patient also revealed that the ringing in her ears still persists 8 months after her last oritavancin dose. This could potentially be a serious adverse event associated with multiple doses of oritavancin.

In addition to our case series, oritavancin has been used clinically in a number of scenarios and also possesses some impressive in vivo data. To date, it has been demonstrated that the single oritavancin dose is convenient for treatment of Gram-positive infections. The SIMPLIFI trial included 302 patients and evaluated different dosing strategies for oritavancin-a one-time dose of $1200 \mathrm{mg}$, a 200-mg daily dose for 37 days, or infrequent dosing which consisted of an 800-mg dose with the option of an additional 400-mg dose on day 5 [17]. The results demonstrated that the one-time front-loading dose of $1200 \mathrm{mg}$ and the infrequent dosing strategy was as efficacious and safe as daily administration in treating ABSSSIs caused by Gram-positive infections, including MRSA. Similar to ABSSSIs, the one-time dose of oritavancin for complicated invasive infections, such as endocarditis and bacteremia, would allow patients to receive adequate treatment before discharge and would ensure adherence.

To date, there are limited data on oritavancin use in serious systemic infections. In the SOLO I trial that evaluated the efficacy and safety of a single dose of oritavancin compared to a 7- to 10-day course of vancomycin, only $3.8 \%$ of patients in the oritavancin arm and $1.9 \%$ of patients in the vancomycin arm had positive blood cultures [18]. Likewise, in the SOLO II trial, which also evaluated oritavancin efficacy and safety versus vancomycin, only $2 \%$ of patients in each the oritavancin and vancomycin arm had positive blood cultures secondary to their ABSSSI infection [19]. In both these pivotal trials, oritavancin was shown to be non-inferior to vancomycin in treatment of ABSSSIs, but there was no mention on outcomes for the few patients who also had bacteremia. These results suggest that more data are needed to determine the efficacy and outcomes of oritavancin in invasive infections due to Gram-positive bacteremia.

A case report published in late 2015 described a 73-year-old male who had vancomycin-resistant Enterococcus faecium prosthetic valve endocarditis that was treated with a prolonged course of oritavancin [20]. In this complicated case, the patient received multiple antibiotics for his recurrent VRE bacteremia, including daptomycin, tigecycline, linezolid, and gentamicin, but these were poorly tolerated with associated anorexia, nausea, thrombocytopenia, hyperlactatemia, and altered mental status. The patient was eventually treated with oritavancin $1200 \mathrm{mg}$ every other day for three doses then once weekly for 6 weeks after susceptibility testing of the VRE isolate showed an oritavancin MIC of $0.5 \mathrm{mg} / \mathrm{L}$. After discontinuation of the other antibiotics and continuation of the oritavancin, the anorexia, altered mental status, and platelet count returned to baseline. Blood cultures remained negative throughout his initial course of oritavancin, but 8 days after completion of oritavancin, his blood cultures again grew VRE. Oritavancin was reinitiated at a dose of $1200 \mathrm{mg}$ twice weekly. The patient underwent an aortic and mitral valve replacement, and the surgical culture from the prosthetic aortic valve grew VRE, and the mitral valve culture had evidence of healing endocarditis. He continued twiceweekly infusions of oritavancin for 10 weeks with no return of positive blood cultures, but at the 10-week follow-up visit he experienced elevated liver function tests (LFTs) and oritavancin was stopped. His LFTs returned to baseline 11 months after oritavancin completion. This case report shows potential for oritavancin therapy in invasive infections, such as VRE endocarditis [20]. This case also emphasizes the need for more studies evaluating different dosing strategies for oritavancin therapy in invasive Gram-positive infections. 
Oritavancin has been used on a case-by-case basis by our ID physicians as an alternative in patients who would otherwise have required long courses of treatment for serious infections, and who may not have completed those courses due to individual circumstances. This medication can potentially have a niche in this difficult-to-treat patient population, and the one-time infusion of oritavancin ensures compliance with treatment. However, it cannot be ignored that the two patients who failed oritavancin therapy had complicated infections such as osteomyelitis and endocarditis, two serious diseases states where a long-acting antibiotic, such as oritavancin, would be desirable. Furthermore, a potential limitation to this case series is the fact that none of the patients received oritavancin monotherapy. Since all the patients received standard of care, and some patients received suppressive oral therapy post-oritavancin dose, it is difficult to say that the clinical cures in this case series are strictly due to oritavancin itself. Moreover, one ethical issue is discharging patients who have potential compliance issues with one dose of oritavancin for an infection requiring many weeks of antibiotics. This could lead to loss of follow-up and inappropriate duration of therapy for a complicated, serious infection. With this in consideration, the ID physicians at our institution felt it was better to give these patients an antibiotic that we knew would last at least a few weeks instead of no treatment at all.

\section{CONCLUSION}

In conclusion, given the limited data available regarding oritavancin therapy in complicated Gram-positive infections, this series suggests promising outcomes in disease states such as $S$. aureus and enterococcal bacteremia. Normally, the standard of care is several weeks of IV antibiotics. Unfortunately, there are situations, such as those described in this report, where delivering this standard of care is not possible. Additional data and literature are needed on the outcomes of patients treated with oritavancin for indications other than ABSSSIs before oritavancin can be routinely recommended.

\section{ACKNOWLEDGEMENTS}

No funding or sponsorship was received for this study or publication of this article. All named authors meet the International Committee of Medical Journal Editors (ICMJE) criteria for authorship for this manuscript, take responsibility for the integrity of the work as a whole, and have given final approval for the version to be published.

Disclosures. Cassie L. Stewart, Jeremy J. Frens, Michelle S. Turner, Jordan R. Smith, and Cynthia B. Snider have no disclosures or conflicts of interest.

Compliance with Ethics Guidelines. This was a retrospective study reviewing chart data therefore no new studies with human or animal subjects were performed by any of the authors. A waiver of informed consent was obtained from the Cone Health Institutional Review Board.

Data Availability. The datasets during and/ or analyzed during the current study are available from the corresponding author on reasonable request.

Open Access. This article is distributed under the terms of the Creative Commons Attribution-NonCommercial 4.0 International License (http://creativecommons.org/licenses/ by-nc/4.0/), which permits any noncommercial use, distribution, and reproduction in any medium, provided you give appropriate credit to the original author(s) and the source, provide a link to the Creative Commons license, and indicate if changes were made.

\section{REFERENCES}

1. Menichetti F. Current and emerging serious Gram-positive infections. Clin Microbiol Infect. 2005;11(Suppl 3):22-8.

2. Sievert DM, Ricks P, Edwards JR, et al. Antimicrobial-resistant pathogens associated with healthcare-associated infections: summary of data 
reported to the National Healthcare Safety Network at the Centers for Disease Control and Prevention, 2009-2010. Infect Control Hosp Epidemiol. 2013;34:1-14.

3. Cosgrove SE, Sakoulas G, Perencevich EN, et al. Comparison of mortality associated with methicillin-resistant and methicillin-susceptible Staphylococcus aureus bacteremia: a meta-analysis. Clin Infect Dis. 2003;36:53-9.

4. van Hal SJ, Jensen SO, Vaska VL, et al. Predictors of mortality in Staphylococcus aureus bacteremia. Clin Microbiol Rev. 2012;25:362-86.

5. Liu C, Bayer A, Cosgrove SE, et al. Clinical practice guidelines by the Infectious Diseases Society of America for the treatment of methicillin-resistant Staphylococcus aureus infections in adults and children. Clin Infect Dis. 2011;52:e18-55.

6. Rybak M, Lomaestro B, Rotschafer JC, et al. Therapeutic monitoring of vancomycin in adult patients: a consensus review of the American Society of Health-System Pharmacists, the Infectious Diseases Society of America, and the Society of Infectious Diseases Pharmacists. Am J Health Syst Pharm. 2009;66:82-98.

7. Tice AD, Rehm SJ, Dalovisio JR, et al. Practice guidelines for outpatient parenteral antimicrobial therapy. IDSA guidelines. Clin Infect Dis. 2004;38:1651-72.

8. Corey GR, Good S, Jiang H, et al. Single-dose oritavancin versus 7-10 days of vancomycin in the treatment of Gram-positive acute bacterial skin and skin structure infections: the SOLO II Noninferiority Study. Clin Infect Dis. 2015;60:254-62.

9. Smith JR, Yim J, Raut A, et al. Oritavancin combinations with beta-lactams against multidrug-resistant Staphylococcus aureus and vancomycin-resistant enterococci. Antimicrob Agents Chemother. 2016;60:2352-8.

10. Rubino CM, Bhavnani SM, Moeck G, et al. Population pharmacokinetic analysis for a single 1,200-milligram dose of oritavancin using data from two pivotal phase 3 clinical trials. Antimicrob Agents Chemother. 2015;59:3365-72.

11. Rubino CM, Van Wart SA, Bhavnani SM, et al. Oritavancin population pharmacokinetics in healthy subjects and patients with complicated skin and skin structure infections or bacteremia. Antimicrob Agents Chemother. 2009;53:4422-8.

12. Mendes RE, Sader HS, Flamm RK, et al. Oritavancin activity against Staphylococcus aureus causing invasive infections in U.S. and European hospitals: a 5-year international surveillance program. Antimicrob Agents Chemother. 2014;58:2921-4.

13. Duncan LR, Sader HS, Flamm RK, et al. Oritavancin in vitro activity against contemporary Staphylococcus aureus isolates responsible for invasive community- and healthcare-associated infections among patients in the United States (2013-2014). Diagn Microbiol Infect Dis. 2016;86:303-6.

14. Morrissey I, Seifert H, Canton R, et al. Activity of oritavancin against methicillin-resistant staphylococci, vancomycin-resistant enterococci and betahaemolytic streptococci collected from western European countries in 2011. J Antimicrob Chemother. 2013;68:164-7.

15. Mendes RE, Woosley LN, Farrell DJ, et al. Oritavancin activity against vancomycin-susceptible and vancomycin-resistant Enterococci with molecularly characterized glycopeptide resistance genes recovered from bacteremic patients, 2009-2010. Antimicrob Agents Chemother. 2012;56:1639-42.

16. Mellor JA, Kingdom J, Cafferkey M, et al. Vancomycin ototoxicity in patients with normal renal function. Br J Audiol. 1984;18:179-80.

17. Dunbar LM, Milata J, McClure T, et al. Comparison of the efficacy and safety of oritavancin front-loaded dosing regimens to daily dosing: an analysis of the SIMPLIFI trial. Antimicrob Agents Chemother. 2011;55:3476-84.

18. Corey GR, Kabler H, Mehra P, et al. Single-dose oritavancin in the treatment of acute bacterial skin infections. N Engl J Med. 2014;370:2180-90.

19. Corey GR, Good S, Jiang H, et al. Single-dose oritavancin versus 7-10 days of vancomycin in the treatment of gram-positive acute bacterial skin and skin structure infections: the SOLO II noninferiority study. Clin Infect Dis. 2015;60:254-62.

20. Johnson JA, Feeney ER, Kubiak DW, et al. Prolonged use of oritavancin for vancomycin-resistant Enterococcus faecium prosthetic valve endocarditis. Open Forum Infect Dis. 2015;2:ofv156. 